QCD Evolution Workshop 2014

International Journal of Modern Physics: Conference Series Vol. 37 (2015) 1560039 (8 pages)

(C) The Authors

DOI: $10.1142 / \mathrm{S} 2010194515600393$

\title{
Observables for Quarks and Gluons Orbital Angular Momentum Distributions
}

\author{
Simonetta Liuti* \\ Physics Department, University of Virginia, \\ 382 McCormick Rd., Charlottesville, Virginia 22904, USA \\ INFN, Laboratori Nazionali di Frascati, \\ Via E. Fermi 40, 00044, Frascati RM, Italy \\ sl4y@virginia.edu \\ Aurore Courtoy \\ IFPA, AGO Department, Université de Liège, \\ Bât. B5, Sart Tilman B-4000 Liège, Belgium \\ aurore.courtoy@ulg.ac.be \\ Gary R. Goldstein \\ Department of Physics and Astronomy, \\ Tufts University, Medford, MA 02155, USA \\ gary.goldstein@tufts.edu \\ J. Osvaldo Gonzalez Hernandez \\ Istituto Nazionale di Fisica Nucleare (INFN) - Sezione di Torino \\ via P. Giuria, 1, 10125 Torino, ITALY, Italy \\ joghdr@gmail.com \\ Abha Rajan \\ Physics Department, University of Virginia, \\ 382 McCormick Rd., Charlottesville, Virginia 22904 - USA \\ ar5xc@virginia.edu
}

Published 25 February 2015

\begin{abstract}
We discuss the observables that have been recently put forth to describe quarks and gluons orbital angular momentum distributions. Starting from a standard parameterization of the energy momentum tensor in QCD one can single out two forms of angular momentum, a so-called kinetic term - Ji decomposition - or a canonical term - Jaffe-Manohar decomposition. Orbital angular momentum has been connected in each decomposition
\end{abstract}

* Speaker

This is an Open Access article published by World Scientific Publishing Company. It is distributed under the terms of the Creative Commons Attribution 3.0 (CC-BY) License. Further distribution of this work is permitted, provided the original work is properly cited. 


\section{S. Liuti et al.}

to a different observable, a Generalized Transverse Momentum Distribution (GTMD), for the canonical term, and a twist three Generalized Parton Distribution (GPD) for the kinetic term. While the latter appears as an azimuthal angular modulation in the longitudinal target spin asymmetry in deeply virtual Compton scattering, due to parity constraints, the GTMD associated with canonical angular momentum cannot be measured in a similar set of experiments.

Keywords: QCD energy momentum tensor; angular momentum sum rule; deeply virtual exclusive experiments.

\section{Introduction}

One of the important challenges for QCD is understanding the angular momentum or spin structure of the nucleon where a fundamental question has recently arisen about developing a unique gauge invariant decomposition of the total quark and gluon angular momenta, $J^{q}$, and $J^{g}$, into their respective spin and orbital components (see Refs. [1, 2] for reviews). Although this issue is yet to be resolved, out of the many possibilities for such a decomposition, emerge two fundamental forms, leading to the so-called kinetic Orbital Angular Momentum (OAM) - also known as Ji decomposition [3] - and the canonical orbital angular momentum Jaffe-Manohar [4] decomposition. The Ji decomposition reads [3],

$$
\frac{1}{2} \Delta \Sigma+L^{q}+J^{g}=\frac{1}{2}
$$

$L^{q}$, the kinetic OAM, is at variance with the canonical OAM, $L_{\text {can }}^{q, g}$ which is defined through the decomposition [4],

$$
\frac{1}{2}=\frac{1}{2} \Delta \Sigma+L_{\mathrm{can}}^{q}+\Delta G+L_{\mathrm{can}}^{g} .
$$

$L_{q}(x)$ and $L_{\text {can }}^{q}(x)$ admit the same Wandzura-Wilczek (WW) part, $L_{q}^{W W}(x)$, while they differ in their genuine twist three contribution $[5,6]$,

$$
\begin{gathered}
L_{q}(x)=L_{q}^{W W}(x)+\bar{L}_{q}(x) \\
L_{\text {can }}^{q}(x)=L_{q}^{W W}(x)+\bar{L}_{\text {can }}^{q}(x) .
\end{gathered}
$$

In the WW limit the two OAM distributions coincide, their differences depend on final state interactions contained in this case in the genuine twist three terms. In particular, $\int d x \bar{L}_{q}(x)=0$, while $\int d x \bar{L}_{\text {can }}^{q}(x) \neq 0$, in general, so that it contributes to the angular momentum sum rule within this specific decomposition.

$L_{q}(x)$ can be defined by extending the Ji sum rule to twist three as $[7,6]$,

$$
\begin{aligned}
\int d x x G_{2}^{q}(x, 0,0) & =\frac{1}{2}\left[-\int d x x\left(H^{q}(x, 0,0)+E^{q}(x, 0,0)\right)+\int d x \widetilde{H}^{q}(x, 0,0)\right] \\
& \rightarrow \int d x x G_{2}^{q}(x, 0,0)=-L^{q}
\end{aligned}
$$


where $G_{2}^{q}$ is a specific Generalized Parton Distribution (GPD) appearing in the parametrization of the quark-quark correlation function at twist three $[7,8,9,10]$ $\left(G_{2}\right.$ was renamed $\widetilde{E}_{2 T}$ in the full classification of GPDs given in Ref.[11]); $H^{q}$, $E^{q}$, and $\widetilde{H}^{q}$ are the twist two GPDs contributing to the observables for Deeply Virtual Compton Scattering (DVCS) processes introduced in [3] (see reviews in Refs. $[12,13])$.

On the other side, canonical OAM is constructed by parametrizing the unintegrated correlation function [4] in the following way [5, 14, 15, 16],

$$
\begin{aligned}
L_{c a n}^{q} & =\left\langle p, \Lambda\left|\int d x^{-} d \mathbf{x}_{\perp} i \psi^{\dagger}(\mathbf{x} \times \nabla)^{3} \psi\right| p, \Lambda\right\rangle \\
& =-\int d x d^{2} k_{T} \frac{k_{T}^{2}}{M^{2}} F_{14}\left(x, 0, k_{T}^{2}, 0,0\right),
\end{aligned}
$$

where $F_{14}$ is a specific GTMD - or an unintegrated over intrinsic- $k_{T}$ GPD - appearing in the decomposition of the vector component of the unintegrated quark-quark correlation function at twist two [11].

The connection of $L_{\text {can }}^{q}$, with kinetic OAM was discussed in several papers [1, 2]. Indeed Eq.(5) provides a plausible, intuituive identification which is inferred from the definition of canonical OAM originally suggested in [4]. Nevertheless, the fact that one can consider matching OAM onto experimental observables, only through a specific off-forward unintegrated parton distribution, or GTMD, entails various complications, from questions on both its factorizability and renormalizability in QCD, to the definition of a deeply virtual scattering process which could be sensitive to $F_{14}$. Such complications are not present for the GPD, $G_{2}$, although there exists no obvious, straightforward partonic interpretation of this twist three quantity.

Notwithstanding the notion that the GPDs that enter Eq. (4) can be observed by measuring specific DVCS asymmetries and cross sections, to validate this relation it is however necessary to identify processes where OAM can be observed directly through the twist three GPD, $G_{2}$. This was done in Ref. [17] where, making use of the expressions from an extensive analysis of DVCS at twist three level performed in $[9,10]$, we were able to single out the helicity amplitudes combinations which contribute to the twist three GPD $G_{2}$, and to connect this structure function to an observable, namely the sin $2 \phi$ modulation in the longitudinal Target Spin Asymme$\operatorname{try}(\mathrm{TSA}), A_{\mathrm{LU}}^{\sin 2 \phi}[17]$. This term has already been measured, and found to be quite substantial at HERMES [18] and CLAS [19]. It is also presently been analyzed at Jefferson Lab [20].

In this contribution we address once more these issues, with the specific goal to provide additional support for pursuing experiments sensitive to both canonical and kinetic OAM. A more profound physical understanding of OAM may emerge only by defining a way to measure it. We discuss whether this goal can be met in either case, $F_{14}$ and $G_{2}$, and which experimental setup would be required. 


\section{S. Liuti et al.}

\section{Parity Constraints}

In Ref. [17] we demonstrated that there was a fundamental reason behind the claim that it was "not known how to extract Wigner distributions or GTMDs from experiments" [14], namely we explained how this inherent difficulty was a consequence of parity constraints on the helicity amplitudes which enter the general cross section formulation $[21,22]$.

Differently from the Transverse Momentum Distributions (TMDs) and the Compton Form Factors (CFFs) which can be extracted from semi-inclusive and deeply virtual exclusive lepton nucleon scattering, GTMDs cannot be obtained from two body scattering processes. In particular, the DVCS process factors into $\gamma^{*}$-quark elastic scattering and two body quark-proton scattering. In such a process, it is always possible to define a Center-of-Mass (CoM) system where the two transverse momenta, $\mathbf{k}_{T}$ and $\boldsymbol{\Delta}_{T}$ cannot be independent from one another (i.e. they belong to a single hadronic scattering plane).

To extract $F_{14}$ from experiment one first writes the helicity amplitudes for the $\gamma^{*} p$ scattering process. The quark-proton scattering helicity amplitudes content of $F_{14}$ was identified as [17],

$$
i \frac{\bar{k}_{T}^{1} \Delta_{2}-\bar{k}_{T}^{2} \Delta_{1}}{M^{2}} F_{14}=A_{++,++}+A_{+-,+-}-A_{-+,-+}-A_{--,--},
$$

where $\bar{k}_{T}=\left(k_{T}+k_{T}^{\prime}\right) / 2$, and we defined, $A_{\Lambda^{\prime} \lambda^{\prime}, \Lambda \lambda}, \Lambda\left(\Lambda^{\prime}\right)$ and $\lambda\left(\lambda^{\prime}\right)$ being the proton and quark initial (final) helicities, respectively. The helicity amplitudes obey the following parity relation,

$$
A_{-\Lambda^{\prime}-\lambda^{\prime},-\Lambda-\lambda}=\eta_{P}(-1)^{\Lambda^{\prime}-\lambda^{\prime}-\Lambda+\lambda} A_{\Lambda^{\prime} \lambda^{\prime}, \Lambda \lambda}^{*},
$$

$\eta_{P}$ being the phase factor accounting for intrinsic parity and spin.

Therefore, for the $F_{14}$ contribution to the nucleon matrix elements to be nonzero, at least one pair of the helicity amplitudes must be imaginary, at variance with the other spin conserving structure functions. While for GPDs and TMDs this would be unphysical (parity violating) in the proton spin non flip case, for GTMDs, by allowing for relative phases among the amplitudes, the combination that forms $F_{14}$ can indeed be imaginary, as one simultaneously moves away from a collinear description, i.e. as $\bar{k}$ and $\Delta$ are let to vary independently from one another. The specific combination of amplitudes giving rise to $F_{14}$ is therefore consistent with parity conservation so long as one gives up the idea of the quark proton scattering occurring in a single hadronic plane. We therefore here acknowledge that it is preferable to use an alternative choice of words to "parity odd" to describe this rather complicated situation.

This does not imply that $F_{14}$ cannot represent OAM. In fact, by observing that,

$$
i \sigma^{i j} \bar{k}_{T i} \Delta_{T j}=\epsilon^{i j k} \Sigma_{k} \bar{k}_{T i} \Delta_{T j}=\boldsymbol{\Sigma} \cdot\left(\overline{\mathbf{k}}_{T} \times \boldsymbol{\Delta}_{T}\right) \equiv \Sigma_{3}\left(\overline{\mathbf{k}}_{T} \times \boldsymbol{\Delta}_{T}\right)_{3},
$$

one sees that because of the action of $\Sigma$ on the proton matrix elements, one has that $i \sigma^{i j} \bar{k}_{T i} \Delta_{T j}$ transforms in an opposite way to helicity, namely to the corresponding structures $G_{14}$, in the GTMD sector, and $\widetilde{H}$ in the GPD sector [17, 11]. 
Differently from helicity, which is promptly observable, the matrix element corresponding to $F_{14}$ is parity even. Although this is the source of the measurability issue for $F_{14}$, it does not interfere with its identification with OAM which is also a parity even quantity.

Summarizing this part, to measure $F_{14}$ and $G_{11}$ and be consistent with the parity transformation properties in QCD one needs to define therefore an additional hadronic plane. Because $F_{14}$ has the kinematic factor for a longitudinally polarized target going to an unpolarized quark and spectator, it is clear that the hadronization process of the active quark will involve unpolarized functions. Also, as GTMDs depend on the momentum transfer, one has to consider exclusive processes, which rules out "dihadron" fragmentation functions. An exclusive process of the type: $\gamma^{*}+p \rightarrow \gamma+\pi^{+}+\pi^{-}+p^{\prime}$ will be required. The 4 -momenta are set as $q+p=$ $q^{\prime}+p_{1}+p_{2}+p^{\prime}$. There are 5 invariants, $s=(p+q)^{2}, t=\left(p^{\prime}-p\right)^{2}, s_{12}=\left(p_{1}+\right.$ $\left.p_{2}\right)^{2}, s_{13}=\left(p_{1}+p^{\prime}\right)^{2}, t_{1}=\left(q-p_{1}\right)^{2}$. All other invariants can be written in terms of these. With this kinematical set of variables one can fix the $k_{T}$ of the incoming quark, as we will elaborate on in future work.

\section{Angular Momentum Distributions}

The recent developments in $[7,5,6,14,16,17]$ allow us to see how both canonical and kinetic OAM can be represented and compared at the density level. Both distributions describe quarks that are displaced from the origin in the transverse plane. For $F_{14}$, the displacement is obtained through Fourier transformation of the quark-quark correlator components defining this structure function [14],

$$
\text { F.T. }\left(-i \frac{\bar{k}_{1} \Delta_{2}-\bar{k}_{2} \Delta_{1}}{M^{2}} F_{14}\left(x, 0, \overline{\mathbf{k}}_{T}, \boldsymbol{\Delta}_{T}\right)\right)=-\frac{1}{M^{2}} \epsilon_{T}^{i j} \bar{k}_{T}^{i} \frac{\partial}{\partial b_{j}} \mathcal{F}_{14}\left(x, 0, \overline{\mathbf{k}}_{T}, \mathbf{b}\right),
$$

where, $\epsilon_{T}^{i j}=\epsilon^{-+i j}$, and,

$$
\mathcal{F}_{14}\left(x, 0, \overline{\mathbf{k}}_{T}, \mathbf{b}\right)=\int \frac{d^{2} \Delta_{T}}{(2 \pi)^{2}} e^{-i \mathbf{b} \cdot \boldsymbol{\Delta}_{T}} F_{14}\left(x, 0, \overline{\mathbf{k}}_{T}, \boldsymbol{\Delta}_{T}\right)
$$

Notice that $\overline{\mathbf{k}}_{T}$ needs to be kept at a fixed value in order to see this displacement, i.e. an integration over $\overline{\mathbf{k}}_{T}$ gives a zero result. This configuration corresponds to OAM generated through circular motion in the $x-y$ plane [14].

For the configuration corresponding to the unintegrated $G_{2} / \widetilde{E}_{2 T}$ (for more details on the specific GTMDs see Refs.[17, 11], the distortion in the transverse plane is described by the Fourier transform of the distribution,

$$
\begin{array}{r}
\text { F.T. }\left[-i \epsilon_{T}^{i j} \frac{\Delta_{j}}{M} \widetilde{E}_{2 T}\left(x, 0, \overline{\mathbf{k}}_{T}, \boldsymbol{\Delta}_{T}\right)\right]=-\frac{1}{M} \epsilon_{T}^{i j} \frac{\partial}{\partial b_{j}} \widetilde{\mathcal{E}}_{2 T}\left(x, 0, \overline{\mathbf{k}}_{T}, \mathbf{b}\right) \\
(i, j=1,2),
\end{array}
$$

where we used the notation of [11]. In this case $\mathbf{k}_{T}$ can be parallel to $\boldsymbol{\Delta}_{T}$, so Eq.(11) gives a non zero result when integrated over $\mathbf{k}_{T}$, and OAM points in the 


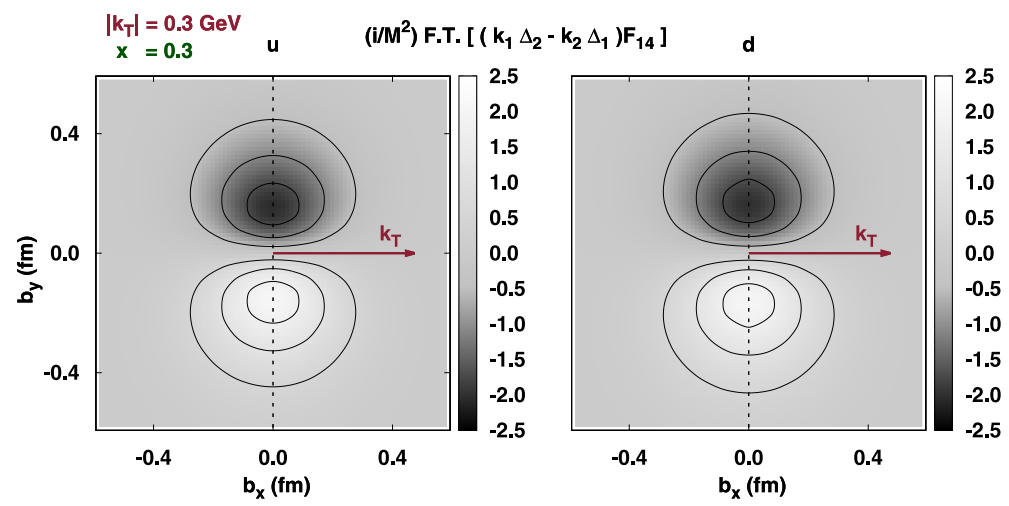

Fig. 1. Fourier transform of the correlator components defining $F_{14}$, Eq.(9) for $x=0.3, k_{1}=0.3$ $\mathrm{GeV}$, and $k_{2}=0$. The $u$ and $d$ quarks contributions are represented on the LHS and RHS, respectively (adapted from Ref. [24]).
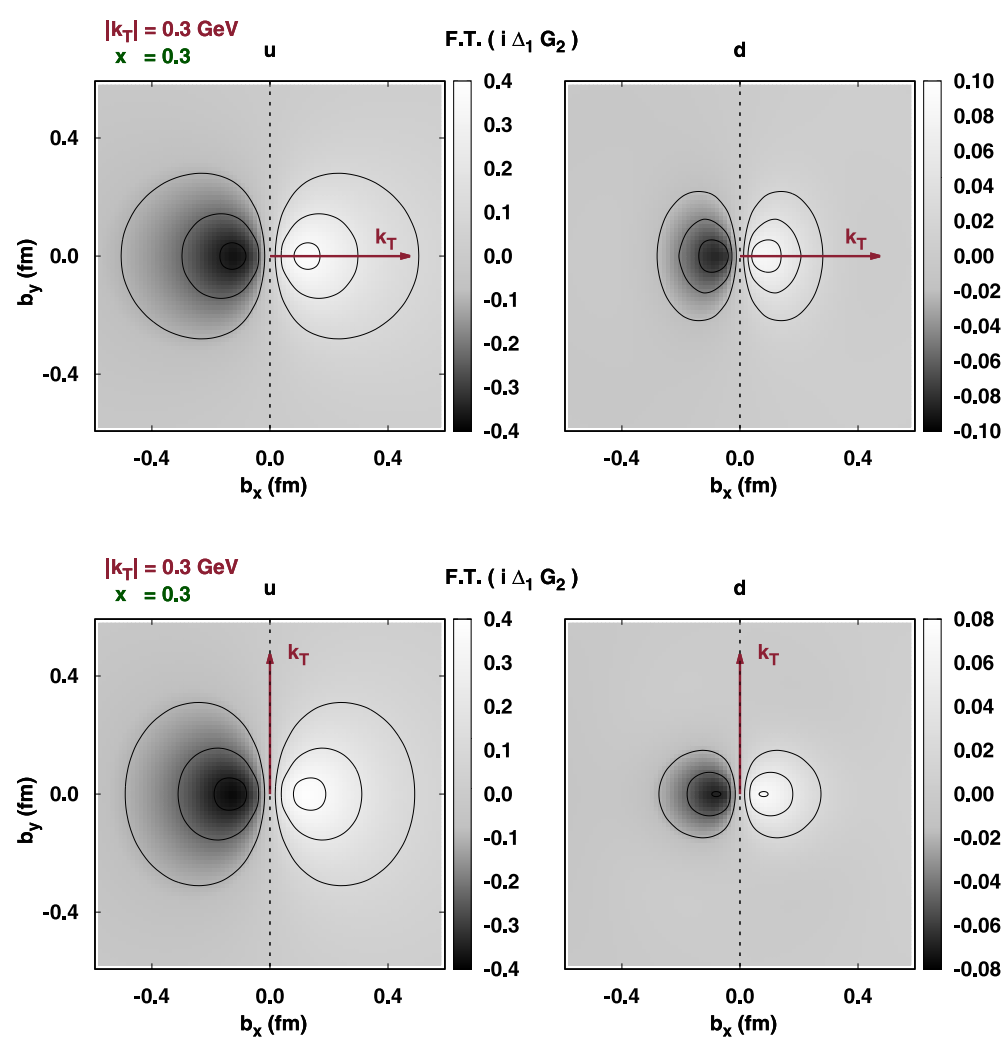

Fig. 2. Fourier transform of the correlator components defining $\widetilde{E}_{2 T}$, Eq. (11) at $x=0.3$, for the components: $k_{1}=0.3 \mathrm{GeV}$, and $k_{2}=0$ (upper panels); $k_{1}=0$, and $k_{2}=0.3 \mathrm{GeV}$ (lower panels). For both the upper and lower panels, the $u$ and $d$ quarks contributions are represented on the LHS and RHS, respectively (adapted from Ref. [24]). 
direction orthogonal to both $S_{L}$ and $\boldsymbol{\Delta}_{T}$, consistently with the representation given in Ref.[23].

In Figure 1 and Figure 2 we show the distributions in the transverse plane corresponding to Eq.(9) and to the $i=2$ component of Eq.(11), respectively. All functions were obtained in the reggeized quark-diquark picture of Refs. [25, 26].

\section{Conclusions}

In conclusion, we have analyzed the issue of observability of both canonical and kinetic OAM. Canonical OAM can be identified with the second moment in $k_{T}$ of the GTMD $F_{14}$, a formal proof that such quantity is related to OAM having been given in Ref. [5]. The observability of OAM is however hampered by the fact that $F_{14}$, and an analogously a GTMD in the axial-vector sector, $G_{11}$, cannot be connected to any of the GPDs and/or TMDs, thus making it challenging to define physical processes which would be sensitive to these quantities. In spite of the fact that non-zero results for $F_{14}$ can be obtained from either models, or by direct calculations on the lattice, a process that selects this quantity has not been yet identified. The physical content of the models, whether these are "perturbative" or arise from "effective field theories" cannot be taken as a proof of the existence of an observable. However, we notice that each of the models explored so far giving a non-zero result for $F_{14}$, carry some remnant of confinement, while only one model calculation that clearly does not have confinement - the quark-target model - (as an "ensemble of free quarks", with no gluon across the vertices) gives zero for $F_{14}$ (see analogous calculation in Ref. [27 ] on $g_{2}$ ). We take this as an indication that the gauge link structure of $F_{14}$ plays a fundamental role, as already suggested in [16] and that, looking at future studies, its connection with the final state interactions implicitly present in the twist three definition of OAM through $G_{2} / \widetilde{E}_{2 T}$ will give key information on the nature of OAM.

Finally, we reiterate that in our analysis, while reinforcing the use of the LF, we give a physical motivation for the fact that $F_{14}$ has not yet been associated to any observable, that goes beyond simply stating the issue $[14,11]$. Our explanation is founded on the transformation properties of the unintegrated correlator under parity which do not allow for the specific combination of helicity amplitudes generating $F_{14}$ to be observed in any given single hadronic plane. This prompts the derivation of an extension of the "master formula" used so far to describe both semi-inclusive and exclusive lepton-proton scattering [21]. At the same time we point out that the transformation property of the matrix element associated with $F_{14}$ is a distinct issue that should not be confused with the observability of the quantity through its decomposition in quark-proton helicity amplitudes. In this respect, $F_{14}$ is consistent with the transformation under parity of OAM.

\section{Acknowledgments}

We benefitted from the lively atmosphere at the workshop, and from discussions with many colleagues, who were also present there. This work was funded in part 
by the Belgian Fund F.R.S.-FNRS via the contract of Chargée de recherches (A.C.), and by U.S. D.O.E. grant DE-FG02-01ER4120 (S.L., A.R.). A.R. is grateful to the Université de Liège for partial funding.

\section{References}

1. M. Wakamatsu, Int. J. Mod. Phys. A 29, 1430012 (2014); Phys. Rev. D 85, 114039 (2012).

2. E. Leader and C. Lorce, Phys. Rept. 541, 163 (2014)

3. X. -D. Ji, Phys. Rev. Lett. 78, 610 (1997).

4. R. L. Jaffe and A. Manohar, Nucl. Phys. B 337, 509 (1990).

5. Y. Hatta, Phys. Lett. B 708,186 (2012).

6. Y. Hatta and S. Yoshida, JHEP 1210, 080 (2012).

7. D. V. Kiptily and M. V. Polyakov, Eur. Phys. J. C 37, 105 (2004).

8. M. Penttinen, M. V. Polyakov, A. G. Shuvaev and M. Strikman, Phys. Lett. B 491, 96 (2000).

9. N. Kivel, M. V. Polyakov and M. Vanderhaeghen, Phys. Rev. D 63, 114014 (2001).

10. A. V. Belitsky, D. Mueller and A. Kirchner, Nucl. Phys. B 629, 323 (2002).

11. S. Meissner, A. Metz and M. Schlegel, JHEP 0908, 056 (2009).

12. X. Ji, Ann. Rev. Nucl. Part. Sci. 54, 413 (2004).

13. A. V. Belitsky and A. V. Radyushkin, Phys. Rept. 418, 1 (2005).

14. C. Lorce and B. Pasquini, Phys. Rev. D 84, 014015 (2011).

15. C. Lorce, B. Pasquini, X. Xiong and F. Yuan, Phys. Rev. D 85, 114006 (2012).

16. M. Burkardt, Phys. Rev. D 88, no. 1, 014014 (2013).

17. A. Courtoy, G. R. Goldstein, J. O. G. Hernandez, S. Liuti and A. Rajan, Phys. Lett. B 731, 141 (2014).

18. A. Airapetian et al. [HERMES Collaboration], JHEP 1006, 019 (2010).

19. S. Chen et al. [CLAS Collaboration], Phys. Rev. Lett. 97, 072002 (2006).

20. S. Pisano and H. Avakian, private communication.

21. M. Diehl and S. Sapeta, Eur. Phys. J. C 41, 515 (2005)

22. P. J. Mulders and R. D. Tangerman, Nucl. Phys. B 461, 197 (1996); [Erratum-ibid. B 484, $538(1997)]$

23. X. Ji, X. Xiong and F. Yuan, Phys. Rev. Lett. 109, 152005 (2012).

24. A. Courtoy, G. R. Goldstein, J. O. G. Hernandez, S. Liuti and A. Rajan, to be submitted.

25. G. R. Goldstein, J. O. Hernandez and S. Liuti, Phys. Rev. D 84, 034007 (2011).

26. J. O. Gonzalez-Hernandez, S. Liuti, G. R. Goldstein and K. Kathuria, Phys. Rev. C 88, 065206 (2013).

27. R. L. Jaffe and X. D. Ji, Phys. Rev. D 43, 724 (1991). 